\title{
Operational interpretations of quantum discord
}

\author{
D. Cavalcanti, ${ }^{1}$ L. Aolita, ${ }^{2}$ S. Boixo, ${ }^{3}$ K. Modi,${ }^{1}$ M. Piani, ${ }^{4}$ and A. Winter ${ }^{1,5}$ \\ ${ }^{1}$ Centre for Quantum Technologies, National University of Singapore, Singapore 117542 \\ ${ }^{2}$ ICFO-Institut de Ciéncies Fotóniques, Mediterranean Technology Park, 08860 Castelldefels (Barcelona), Spain \\ ${ }^{3}$ Institute for Quantum Information, California Institute of Technology, Pasadena, California 91125, USA \\ ${ }^{4}$ Institute for Quantum Computing and Department of Physics and Astronomy, University of Waterloo, Waterloo, Ontario, Canada, N2L 3G1 \\ ${ }^{5}$ Department of Mathematics, University of Bristol, Bristol BS8 1TW, United Kingdom
}

(Received 25 August 2010; published 31 March 2011)

\begin{abstract}
Quantum discord quantifies nonclassical correlations beyond the standard classification of quantum states into entangled and unentangled. Although it has received considerable attention, it still lacks any precise interpretation in terms of some protocol in which quantum features are relevant. Here we give quantum discord its first information-theoretic operational meaning in terms of entanglement consumption in an extended quantum-statemerging protocol. We further relate the asymmetry of quantum discord with the performance imbalance in quantum state merging and dense coding.
\end{abstract}

DOI: 10.1088/PhysRevA.83.032324

PACS number(s): 03.67.Ac

\section{INTRODUCTION}

The study of quantum correlations has mostly focused on entanglement [1]. This is because entanglement has been identified as a key ingredient in quantum-information processing, allowing performance of a number of tasks that are either impossible to realize or less efficient with only classical resources available. However, entanglement does not account for all the nonclassical properties of quantum correlations. Zurek [2] (see also [3,4]) identified quantum discord (QD) as a feature of quantum correlations that encapsulates entanglement but also goes further, as it is also present even in separable states. Over the past decade, QD has been the focus of several theoretical and experimental studies addressing its formal characterization [5,6], its behavior under dynamic processes [6,7], and its connection with quantum computation [8] and quantum phase transitions [9].

QD was initially introduced within the context of the quantum measurement problem [4], and afterward, interpretations in terms of the difference in performance of quantum and classical Maxwell demons were given [10]. Nevertheless, many in the quantum-information community have remained skeptical toward QD as an information-theoretic quantifier. This is because QD has no clear operational interpretation within this framework. That is, we lack an informationtheoretic task for which the QD provides a quantitative measure regarding the performance of the task. Thus, without this kind of operational interpretation, QD is very often considered simply a "quantumness" parameter.

In this paper we give quantum discord its long-sought operational interpretation. We relate QD to state merging (SM) [11], a well-known task in quantum information. In SM a tripartite pure state is considered, i.e., Alice $(A)$, Bob $(B)$, and Charlie $(C)$ share (many copies of) a pure state $\psi_{A B C}$. The goal in the task is that $A$ transfers her part of the state to $B$, $\psi_{A B C} \rightarrow \psi_{B^{\prime} B C}$ (see Fig. 1), by using classical communication and shared entanglement. Here we show that the minimal total entanglement consumed in a process we call "extended state merging" (ESM) from $A$ to $B$ is exactly equal to the QD between $A$ and $C$ (with measurements on $C$ ). We further unravel a connection between QD to another well-known protocol in quantum-information processing: dense coding (DC) [12]. DC is a task that uses pre-established quantum correlations to send classical messages more efficiently than by classical means.

\section{ENTROPIES, CONDITIONAL ENTROPIES AND COHERENT INFORMATION}

We focus on the finite-dimensional case with the three parties, $A, B$, and $C$, sharing a pure state $\psi_{A B C}$. All bipartite and single-party states are obtained by taking the appropriate partial traces of $\psi_{A B C}$. The quantum (von Neumann) entropy of a state $\rho$ is defined as $S(\rho)=-\operatorname{Tr} \rho \log _{2} \rho$. It is the generalization to the quantum domain of the classical (Shannon) entropy of a probability distribution $\left\{p_{i}\right\}$, given by $H\left(\left\{p_{i}\right\}\right)=-\sum_{i} p_{i} \log _{2} p_{i}$. We write $S(X)$ to denote the entropy of the reduced state $\rho_{X}$. Similarly, we write $H(a)$ to denote the Shannon entropy of a classical random variable $a$ distributed according to some probability distribution $\left\{p_{i}^{a}\right\}$. The latter may be the marginal probability distribution $p_{i}^{a}=\sum_{j} p_{i j}^{a b}$ of a bivariate (in general, multivariate) probability distribution $\left\{p_{i j}^{a b}\right\}$ of two classical random variables $a$ and $b$.

For a bipartite system $A B$, the quantum (von Neumann) conditional entropy is defined as $S(A \mid B):=S(A B)-S(B)$ [13]. It is the quantum version of the classical (Shannon) conditional entropy $H(a \mid b):=H(a, b)-H(b)$. Note that both are asymmetric quantities. $H(a \mid b)$ measures how much uncertainty is left, on average, regarding the value of $a$ given the value of $b$. It can be written as

$$
H(a \mid b)=\sum_{j} p_{j}^{b} H(a \mid b=j),
$$

where $H(a \mid b=j)$ is the entropy of the conditional probability distribution $p_{i \mid b=j}^{a}:=p_{i j}^{a b} / p_{j}^{b}$. It has a clear operational interpretation as the amount of classical information that $A$ has to give - on average - to $B$, who knows the value of $b$, so that the latter gains full knowledge also of the value of $a$ [14]. Given this interpretation for $H(a \mid b)$, it is always non-negative. 

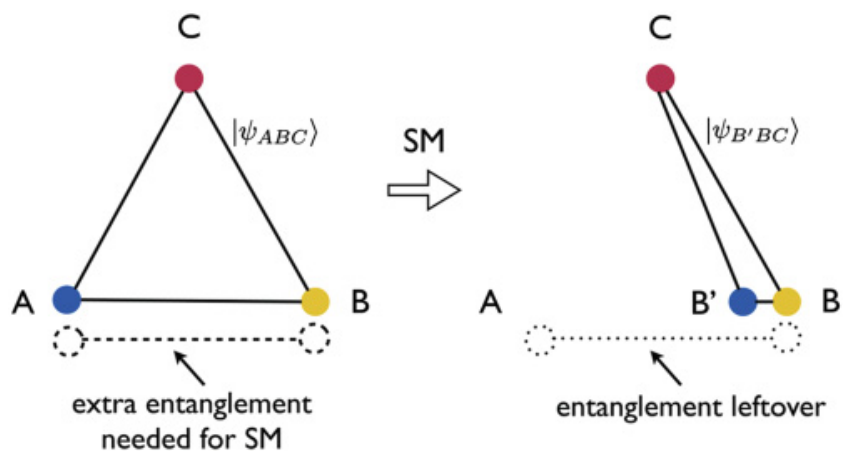

FIG. 1. (Color online) Starting from a tripartite state $\psi_{A B C}$, the goal of SM is to transfer Alice's (A) part of the state to Bob (B), possibly using some extra entanglement or having some entanglement left over. The total entanglement consumption in this process is equal to $D(A \mid C)$ [see Eq. (4)].

However, the situation changes drastically for quantum states, because $S(A \mid B)$ can take negative values, e.g., for pure entangled states. This fact was, for a long time, an obstacle to an operational interpretation of $S(A \mid B)$. Nevertheless, its opposite was identified as an important quantity in the context of quantum information and was even given a name of its own, coherent information, $I(A\rangle B):=-S(A \mid B)$. Coherent information was originally introduced to measure the amount of quantum information conveyable by a quantum channel [15]. Given that it is always nonpositive in the classical case, one may say that it is a purely quantum quantity.

\section{QUANTUM DISCORD}

A remedy to negative quantum conditional entropy is to generalize the classical conditional entropy to quantum using Eq. (1), as was done in [3,4] by defining $S\left(A \mid B_{c}\right):=$ $\min _{\left\{N_{j}\right\}} \sum_{j} p_{j}^{B} S(A \mid B=j)$, where the minimization is over generalized measurements $\left\{N_{j}\right\}^{1}$, with $N_{j} \geqslant 0$ for all $j$ and $\sum_{j} N_{j}=\mathbb{1}_{B}$. We also have $S(A \mid B=j)=S\left(\rho_{A \mid j}\right)$, where $\rho_{A \mid j}=\operatorname{Tr}_{B}\left(\mathbb{1}_{A} \otimes N_{B}^{j} \rho_{A B}\right) / p_{j}^{B}$ with $p_{j}^{B}=\operatorname{Tr}\left(\mathbb{1}_{A} \otimes N_{B}^{j} \rho_{A B}\right)$. $S\left(A \mid B_{c}\right)$ is always non-negative and can also be thought of as a measure of the uncertainty left about $A$ given that $B$ has been measured. For classical systems both $S(A \mid B)$ and $S\left(A \mid B_{c}\right)$ coincide with the classical conditional entropy, but in general, $S\left(A \mid B_{c}\right)$ is larger than, or equal to $S(A \mid B)$. The difference in these two quantities is indeed the definition of the quantum discord with measurements on $B$ [4],

$$
D(A \mid B):=S\left(A \mid B_{c}\right)-S(A \mid B) .
$$

QD can be seen as the gap between the standard measure for total correlations present in a quantum state $\rho_{A B}$, given by quantum mutual information $I(A: B):=S(A)-S(A \mid B)$ [16], and the Henderson-Vedral measure of classical correlations $I\left(A: B_{c}\right):=S(A)-S\left(A \mid B_{c}\right)$ [3]. As $D(A \mid B)=$

\footnotetext{
${ }^{1}$ In [4] the generalized measurement were actually restricted to complete von Neumann measurements.
}

$I(A: B)-I\left(A: B_{c}\right)$, the QD can be considered a (asymmetric) quantifier of nonclassical correlations present in a quantum state. We refer to $D(X \mid Y)$ as the "discord of $X Y$ with measurements on $Y$."

\section{STATE MERGING AND ENTANGLEMENT CONSUMPTION}

A fully convincing operational interpretation of quantum conditional entropy and coherent information was given with the introduction of the task of quantum state merging (SM) [11]. SM, say from $A$ to $B$, is a process by which $A$ and $B$ transfer $A$ 's part of the state to $B$, maintaining the coherence with a reference system $C$. $A$ and $B$ both know the state they share, and they can apply arbitrary local operations coordinated by classical communication (LOCC). By acting on $n$ copies of $\psi_{A B C}$, their goal is to end up with a state close to $\psi_{B^{\prime} B C}^{\otimes n}$, such that the subsystem $B^{\prime}$ is in Bob's hands and plays in the new state exactly the same role as $A$ played in the old one. Errors are allowed, but they must vanish in the limit $n \rightarrow \infty$. To achieve their goal, $A$ and $B$ are allowed to use extra, pre-established two-qubit maximally entangled pairs (ebits), but these constitute a valuable resource they must pay for. It turns out that the value of $S(A \mid B)$ quantifies exactly the optimal amount_-per copy of the state-of ebits spent in the process. A positive value means that entanglement must be consumed, while a negative amount means not only that no extra entanglement is needed, but also that $A$ and $B$ retain $-S(A \mid B)=I(A\rangle B)$ ebits per copy merged. (See Fig. 1 for an illustration of SM.)

A useful way to think of the role played by the conditional entropy in SM is to imagine a hypothetic entanglement bank in which $A$ and $B$ possess a joint account: the entanglement balance after merging-in ebits, per copy merged-is given precisely by $-S(A \mid B)$. When $S(A \mid B) \geqslant 0, A$ and $B$ have to withdraw $S(A \mid B)$ from their account to perform SM. On the other hand, when $S(A \mid B)<0$, then the process can be completed without any withdrawing. Moreover, after merging they end up sharing $I(A\rangle B)=-S(A \mid B)$ extra ebits of entanglement, which they deposit in their account for future use.

At the end of this process, the only correlations between $A$ and $B$ are those present in the bank account. In particular, there is no additional entanglement left between $A$ and $B$. Given this, the bank-account picture suggests to consider a more comprehensive balance that also takes into account the entanglement "lost" in the process. Indeed, coherent information is positive only if the state is entangled, and while $A$ and $B$ may end up with "leftover" Bell pairs after SM, they no longer share the starting entangled states. Thus, it is useful and sensible to define the total entanglement consumption as

$$
\Gamma(A\rangle B):=E_{F}(A: B)+S(A \mid B),
$$

where $E_{F}(A: B):=\min _{\left\{p_{i}, \psi_{i}^{A B}\right\}} \sum_{i} p_{i} S\left(\operatorname{Tr}_{A}\left(\psi_{i}^{A B}\right)\right)$ is the entanglement of formation (EOF) of $\rho_{A B}$, with the minimum taken over pure-state ensembles $\left\{p_{i}, \psi_{i}^{A B}\right\}$ for $\rho_{A B}$ [17]. EOF quantifies the minimum amount of pure-state entanglement that $A$ and $B$ need to consume to create $\rho_{A B}$ by LOCC with strategies where each pure-state member of the ensemble forming $\rho_{A B}$ is prepared independently. Thus, $\Gamma$ quantifies the 
total entanglement consumed in SM, by taking into account the amount of entanglement $A$ and $B$ would have needed to prepare $\rho_{A B}$ by LOCC — and "lost" during SM-plus the amount of entanglement used by the process of SM itself. In order to give a more precise operational interpretation, we consider a two-step process. In the first stage, Alice and Bob prepare the state $\rho_{A B}$. To this aim they have to share classical information and potentially use some other local ancillas. We demand that in order to end up sharing $\rho_{A B}$ and not some larger state, after preparing the state and before the merging, they remove all ancillas. Then Eq. (3) indeed characterizes the entanglement cost of a two-stage process that we call extended state merging (ESM): (i) state preparation through the (possibly nonoptimal - see the Regularization section below) protocol described previously and (ii) merging.

\section{OPERATIONAL INTERPRETATIONS OF QUANTUM DISCORD}

\section{A. Quantum discord and extended-state merging}

Now we are in the position to give QD an operational interpretation. In Appendix A we prove the following:

$$
D(A \mid C)=\Gamma(A\rangle B) .
$$

This equation says that QD between $C$ and $A$ with measurements on $C$ is equal to the total entanglement consumption in ESM from $A$ to $B$. To the best of our knowledge, this yields the first information-theoretic scenario where the value of QD provides concrete quantitative information about a task's performance or cost.

\section{B. Asymmetry of quantum discord}

One immediate exercise of the last equation is to give meaning to the asymmetry of $\mathrm{QD}$, that is, the fact that in general, $D(A \mid C) \neq D(C \mid A)$. Thanks to Eq. (4) we can interpret the asymmetry of discord as the difference in the costs of ESM for $A$ versus $C$ to send their parts of the state to $B$, i.e.,

$$
D(A \mid C)-D(C \mid A)=\Gamma(A\rangle B)-\Gamma(C\rangle B) .
$$

\section{Quantum discord and dense coding}

Coherent information also describes the usefulness of a quantum state $\rho_{A B}$ as a resource for dense coding (DC) [12]. DC - say from a sender $A$ to a receiver $B$, initially sharing $\rho_{A B}$-is a procedure by which $A$ is able, by sending her subsystem to $B$, to transmit more classical information than she could if the system was classical, i.e., the maximal rate of classical information transmission per copy of $\rho_{A B}$ used can be larger. If $A$ 's encoding is done by unitary rotations, the correction to the classical capacity that she could achieve by sending a classical system with dimension equal to that of her subsystem $d_{A}$ is exactly the coherent information $I(A>B)$ [18-21]. In the most general DC scenario [18,19,21], $A$ encodes her message by means of general quantum operations $\Lambda_{A}: M_{d_{A}} \rightarrow M_{d_{A}^{\prime}}$, where $d_{A}$ is the dimension of the original subsystem of $A$, while $d_{A}^{\prime}$ is the dimension of the subsystem sent to $B$, and $M_{d}$ denotes the set of $d \times d$ complex matrices. If the encoding is applied at the level of single copies of the shared state $\rho_{A B}$, the DC single-copy capacity can be achieved by a unitary encoding after a preprocessing operation whose aim is exactly that of increasing coherent information. More precisely, the capacity is equal to $\left.\left.\chi_{\mathrm{DC}}(A\rangle B\right):=\log _{2} d_{A}^{\prime}+\max _{\Lambda_{A}} I\left(A^{\prime}\right\rangle B\right)$, where the maximization is over all quantum operations with output dimension $d_{A}^{\prime}$ and $\left.I\left(A^{\prime}\right\rangle B\right)$ is the coherent information of $\left(\Lambda_{A} \otimes \mathbb{1}_{B}\right)\left[\rho_{A B}\right]$. This capacity depends on the output dimension $d_{A}^{\prime}$, but, given that $\log _{2} d_{A}^{\prime}$ can be considered as a classical contribution, one can focus on the quantum advantage of DC,

$$
\left.\left.\Delta_{\mathrm{DC}}(A\rangle B\right):=\max _{\Lambda_{A}} I\left(A^{\prime}\right\rangle B\right)
$$

The maximization above has no restriction on the output dimension, which nevertheless can be taken to be less than or equal to $d_{A}^{2}$ [21]. The maximization over $\Lambda_{A}$ ensures that the coherent information of the preprocessed state is non-negative.

In Appendix B we prove the following connection between QD and DC:

$$
\left.\left.D(A \mid C)-D(B \mid C)=\Delta_{\mathrm{DC}}(C\rangle A\right)-\Delta_{\mathrm{DC}}(C\rangle B\right) .
$$

Note that if $C$ sends subsystems with the same dimension to $A$ and $B$ (in particular, a dimension large enough to achieve the quantum advantage of DC with both receivers), this difference can be written as $D(A \mid C)-D(B \mid C)=$ $\chi_{\mathrm{DC}}(C>A)-\chi_{\mathrm{DC}}(C>B)$, i.e., in terms of the DC capacity itself.

Equation (7) gives an operational meaning in terms of performance to the differences in QD: the difference in the QD of $A C$ and $B C$, both measured by $C$, is the same as the difference in the DC capacity from $C$ to either $A$ or $B$. The same difference in QD can be related to the coherent information, as can be seen using Eq. (4) twice: $D(A \mid C)-D(B \mid C)=I(A\rangle C)-$ $I(B\rangle C)=I(C\rangle A)$. Or, for measurements on different parties, $D(C \mid A)-D(C \mid B)=\Gamma(C\rangle B)-\Gamma(C\rangle A)$.

\section{REGULARIZATION}

All the relations we have found, although already meaningful in the form above, can be cast in their regularized version so that they become, in the case of ESM, more consistent from an operational and information-theoretic point of view. To do so we note that the minimal amount of ebits needed to create $\rho_{A B}$ over all possible LOCC strategies is given by the entanglement cost $E_{C}(A: B)=\lim _{n \rightarrow \infty} \frac{1}{n} E_{F}(A: B)_{\rho_{A B}^{\otimes n}}$ [22]. We can then define the asymptotic total entanglement consumption of ESM as the regularized version of Eq. (3), i.e., as $\left.\Gamma^{\infty}(A\rangle B\right):=$ $\left.\lim _{n \rightarrow \infty} \Gamma(A\rangle B\right)_{\rho_{A B}^{\otimes n}} / n=E_{C}(A: B)+S(A \mid B)$, having used the additivity of conditional entropy. As ESM is itself an asymptotic process, the regularized total cost $\Gamma^{\infty}$ is a quantity better motivated than the unregularized $\Gamma$ from an operational and information-theoretic point of view. It is worth remarking that both $\Gamma$ and $\Gamma^{\infty}$ are non-negative, because coherent information is a lower bound on distillable entanglement [23] and therefore on entanglement cost. From Eq. (4) we have that $\left.D^{\infty}(A \mid C)=\Gamma^{\infty}(A\rangle B\right)$ 


\section{CONCLUSION}

We have seen that the QD is intimately related to the tasks of ESM and DC. For a pure tripartite state, the QD reveals the entanglement consumption in ESM and in which direction more classical information can be sent through DC. Moreover, the asymmetry of the QD can be given an operational interpretation, since it matches the asymmetry of the tasks to which we have related it, ESM and DC, which are inherently unidirectional.

Finally, a recent paper has unraveled a different connection between QD and SM [24]. There it was observed that the right-hand side of (2) can be interpreted as the difference in quantum-communication costs between performing SM with a partially measured version of $\rho_{A B}$ (first term) and with $\rho_{A B}$ (second term) directly. Such an interpretation of QD regards a relation between different states, one obtained from the other via measurement, while the one presented here refers to just one state (and its purification). On the other hand, since QD can be expressed also as the difference in mutual information between such two states [see the paragraph after Eq. (2)], an approach similar to that of [24] can lead to interpretations in terms of quantum locking [25,26] and correlations erasure [16].

\section{ACKNOWLEDGMENTS}

We thank A. Acín, C. Bény, J. Calsamiglia, C. Caves, A. Datta, M. Gu, and V. Vedral for valuable comments. This work was supported by the National Research Foundation, the Ministry of Education of Singapore, the Spanish "Juan de la Cierva" Programme, NSERC, QuantumWorks, Ontario Centres of Excellence, the Royal Society, UK EPSRC, and the European Commission.

\section{APPENDIX A: PROOF OF EQ. (4)}

We start by recalling the Koashi-Winter monogamy relation [27] for quantum correlations within a pure tripartite state $\psi_{A B C}$ :

$$
S(B)=E_{F}(A: B)+I\left(B: C_{c}\right) .
$$

This, together with the definition of $I\left(B: C_{c}\right)$, implies that

$$
E_{F}(A: B)=S\left(B \mid C_{c}\right)=S\left(A \mid C_{c}\right),
$$

which we can substitute in the definition of $D(A \mid C)$ to get [28]

$$
D(A \mid C)=E_{F}(A: B)-S(A \mid C) .
$$

Now, note that $S(A \mid C)=S(A C)-S(C)$, and since $\psi_{A B C}$ is a pure state, we have $S(A C)=S(B)$ and $S(C)=S(A B)$. Hence $S(A \mid C)=S(B)-S(A B)=-S(A \mid B)$, so that

$$
\left.D(A \mid C)=E_{F}(A: B)+S(A \mid B)=\Gamma(A\rangle B\right) .
$$

\section{APPENDIX B: PROOF OF EQ. (7)}

A monogamy equality similar to Eq. (A1) with regard to DC was given in [21]:

$$
\left.S(A)=E_{P}(A: C)+\Delta_{\mathrm{DC}}(B\rangle A\right),
$$

where $E_{P}$ is the entanglement of purification, defined as [29] $E_{P}(A: C):=\min _{\psi_{A A^{\prime} C C^{\prime}}} S\left(\operatorname{Tr}_{C C^{\prime}}\left(\psi_{A A^{\prime} C C^{\prime}}\right)\right)$, with the minimum taken over all pure states $\psi_{A A^{\prime} C C^{\prime}}$ such that $\operatorname{Tr}_{A^{\prime} C^{\prime}}\left(\psi_{A A^{\prime} C C^{\prime}}\right)=\rho_{A C}$. Using the fact that for a tripartite pure state $I(A\rangle C)=S(C)-S(B)$, and expressing $S(B)$ according to (B1), from (4) one obtains $D(A \mid C)=S(C)-$ $\left.\Delta_{\mathrm{DC}}(C\rangle B\right)-\left[E_{P}(A: B)-E_{F}(A: B)\right]$. By applying this equivalence twice one gets

$$
\left.\left.D(A \mid C)-D(B \mid C)=\Delta_{\mathrm{DC}}(C\rangle A\right)-\Delta_{\mathrm{DC}}(C\rangle B\right) .
$$

[1] R. Horodecki, P. Horodecki, M. Horodecki, and K. Horodecki, Rev. Mod. Phys. 81, 865 (2009).

[2] W. H. Zurek, Ann. Phys. (Berlin) 9, 855 (2000).

[3] L. Henderson and V. Vedral, J. Phys. A: Math. Gen. 34, 6899 (2001).

[4] H. Ollivier and W. H. Zurek, Phys. Rev. Lett. 88, 017901 (2001).

[5] M. Piani, P. Horodecki, and R. Horodecki, Phys. Rev. Lett. 100, 090502 (2008); C. A. Rodriguez-Rosario, K. Modi, A.-M. Kuah, A. Shaji, and E. C. G. Sudarshan, J. Phys. A: Math. Theor. 41, 205301 (2008); K. Modi, T. Paterek, W. Son, V. Vedral, and M. Williamson, Phys. Rev. Lett. 104, 080501 (2010); P. Giorda and M. G. A. Paris, ibid. 105, 020503 (2010); G. Adesso and A. Datta, ibid. 105, 030501 (2010); B. Dakić, V. Vedral, and C. Brukner, ibid. 105, 190502 (2010).

[6] A. Ferraro, L. Aolita, D. Cavalcanti, F. M. Cucchietti, and A. Acín, Phys. Rev. A 81, 052318 (2010).

[7] T. Werlang, S. Souza, F. F. Fanchini, and C. J. Villas Boas, Phys. Rev. A 80, 024103 (2009); J. Maziero, L. C. Celeri, R. M. Serra, and V. Vedral, ibid. 80, 044102 (2009); L. Mazzola, J. Piilo, and S. Maniscalco, Phys. Rev. Lett. 104, 200401 (2010); F. F. Fanchini, T. Werlang, C. A. Brasil, L. G. E. Arruda, and A. O. Caldeira, Phys. Rev. A 81, 052107 (2010); J.-S. Xu, X.-Y. Xu,
C.-F. Li, C.-J. Zhang, X.-B. Zou, and G.-C. Guo, Nat. Commun. 1, 7 (2010).

[8] A. Datta, A. Shaji, and C. M. Caves, Phys. Rev. Lett. 100, 050502 (2008); B. P. Lanyon, M. Barbieri, M. P. Almeida, and A. G. White, ibid. 101, 200501 (2008).

[9] M. S. Sarandy, Phys. Rev. A 80, 022108 (2009); T. Werlang, C. Trippe, G. A. P. Ribeiro, and G. Rigolin, Phys. Rev. Lett. 105, 095702 (2010).

[10] W. H. Zurek, Phys. Rev. A 67, 012320 (2003); A. Brodutch and D. R. Terno, ibid. 81, 062103 (2010).

[11] M. Horodecki, J. Oppenheim, and A. Winter, Nature 436, 673 (2005).

[12] C. H. Bennett and S. J. Wiesner, Phys. Rev. Lett. 69, 2881(1992).

[13] M. A. Nielsen and I. L. Chuang, Quantum Computation and Quantum Information (Cambridge University Press, Cambridge, UK, 2000).

[14] D. Slepian and J. K. Wolf, IEEE Trans. Inf. Theory 19, 461 (1971).

[15] B. Schumacher and M. A. Nielsen, Phys. Rev. A 54, 2629 (1996).

[16] B. Groisman, S. Popescu, and A. Winter, Phys. Rev. A 72, 032317 (2005).

[17] C. H. Bennett, D. P. DiVincenzo, J. A. Smolin, and W. K. Wootters, Phys. Rev. A 54, 3824 (1996). 
[18] M. Horodecki, P. Horodecki, R. Horodecki, D. Leung, and B. Terhal, Quantum Inf. Comput. 1, 70 (2001).

[19] A. Winter, J. Math. Phys. 43, 4341 (2002).

[20] D. Bruß, G. M. D’Ariano, M. Lewenstein, C. Macchiavello, A. Sen(De), and U. Sen, Phys. Rev. Lett. 93, 210501 (2004).

[21] M. Horodecki and M. Piani, e-print arXiv:quant-ph/0701134.

[22] C. H. Bennett, D. P. DiVincenzo, J. A. Smolin, and W. K. Wootters, Phys. Rev. A 54, 3824 (1996).

[23] I. Devetak and A. Winter, Proc. R. Soc. London, Ser. A 461, 207 (2005).

[24] V. Madhok and A. Datta, Phys. Rev. A 83, 032323 (2011).
[25] D. P. DiVincenzo, M. Horodecki, D. W. Leung, J. A. Smolin, and B. M. Terhal, Phys. Rev. Lett. 92, 067902 (2004); P. Hayden, D. Leung, P. W. Shor, and A. Winter, Commun. Math. Phys. 250, 371 (2004).

[26] S. Boixo et al. (in preparation 2011).

[27] M. Koashi and A. Winter, Phys. Rev. A 69, 022309 (2004).

[28] F. F. Fanchini, M. F. Cornelio, M. C. de Oliveira, and A. O. Caldeira, e-print arXiv:1006.2460v1.

[29] B. M. Terhal, M. Horodecki, D. P. DiVincenzo, and D. Leung, J. Math Phys. 43, 4286 (2002). 